\title{
Differences in the Most Demanding Scenarios of Basketball Match-Play between Game Quarters and Playing Positions in Professional Players
}

\author{
Franc García ${ }^{1,2}$, Hugo Salazar², Jordan L. Fox ${ }^{3,4}$ \\ Affiliations: 'Futbol Club Barcelona, Sports Performance Area, Barcelona, Spain, ${ }^{2}$ University of the Basque Country (UPV/EHU), Physical Activity \\ and Sport Department, Vitoria-Gasteiz, Spain, ${ }^{3}$ Central Queensland University, School of Health, Medical, and Applied Sciences, Rockhampton, \\ QLD, Australia, ${ }^{4}$ Central Queensland University, Human Exercise and Training Laboratory, Rockhampton, QLD, Australia
}

Correspondence: F. García, Avinguda Onze de Setembre, s/n, 08970 Ciutat Esportiva Joan Gamper, Sant Joan Despí, Barcelona, Spain. E-mail: francgarciagarrido@gmail.com

\begin{abstract}
The purpose of this study was to compare the most demanding scenarios (MDS) encountered by professional basketball players across game quarters and playing positions during official match-play. Ten professional basketball players were monitored during 11 matches using a local positioning system. Peak physical demands were measured via total distance, distance $>18$ and $>21 \mathrm{~km} \cdot \mathrm{h}^{-1}$, number of sprints $>18$ and $>21 \mathrm{~km} \cdot \mathrm{h}^{-1}$, and number of accelerations and decelerations $>2$ and $>3 \mathrm{~m} \cdot \mathrm{s}^{-2}$ captured over 30,60,120, 180, and 300-s rolling averages. Linear mixed models and effect sizes (ES) were used to compare MDS encountered between game quarters and playing positions. Between Quarters 1 and 2, there was a reduction in the total distance $(E S=0.64-1.39)$ for all playing groups along with a reduction in distance $>21 \mathrm{~km} \cdot \mathrm{h}^{-1}$ in centres $(E S=0.77-0.81)$ and a reduction in accelerations and decelerations $>2 \mathrm{~m} \cdot \mathrm{s}^{-2}$ in guards $(E S=0.66-0.78$ ) across longer sample periods $(180-300-\mathrm{s})$. Between Quarters 1 and 4 , reductions in the total distance were evident for forwards and centres ( $E S=0.64-0.91$ ) as well as distance and sprints $>21 \mathrm{~km} \cdot \mathrm{h}^{-1}$ in centres ( $E S=0.64-0.97$ ). Regarding positional differences, guards and forwards covered a higher total distance than centres across most quarters and sample periods ( $E S=0.22-1.44$ ). Our data suggest a decrease in MDS with game progression in basketball. In addition, MDS appear to be duration-specific and position-dependent in basketball. Therefore, practitioners should consider these differences in MDS based on game quarters and positional demands to optimise individual and team performance.
\end{abstract}

Keywords: team sport, local positioning system, load, player monitoring, worst-case scenarios

@MJSSMontenegro

THE MOST DEMANDING SCENARIOS OF BASKETBALL MATCH-PLAY

http://mjssm.me/?sekcija=article\&artid=227

Cite this article: García, F., Salazar, H., \& Fox, J.L. (2022).Differences in the Most Demanding Scenarios of Basketball Match-Play between Game Quarters and Playing Positions in Professional Players. Montenegrin Journal of Sports Science and Medicine, 11 (1), 15-28. https://doi.org/10.26773/mjssm.220302

\section{Introduction}

Basketball is an intermittent, indoor court-based team sport where high-intensity movements, such as changes of di- rection, accelerations, decelerations, and jumps, are completed amongst periods of rest (Narazaki et al., 2009; Stojanović et al., 2018). Due to the demanding nature of basketball, mon-

Received: 7 June 2021 | Accepted after revision: 7 July 2021 | Early access publication date: 15 August 2021 | Final publication date: 1 March 2022 (c) 2022 by the author(s). License MSA, Podgorica, Montenegro. This article is an open access article distributed under the terms and conditions of the Creative Commons Attribution (CC BY).

Conflict of interest: None declared. 
itoring the physical (external load) and physiological (internal load) demands encountered by players is of critical importance for practitioners in order to promote positive performance-based training adaptations which leave players well-prepared for official games (Sansone et al., 2020). External load is particularly important from a practical perspective as practitioners can directly manipulate it to bring about the desired adaptations in players. Consequently, a detailed understanding of the external loads encountered by players during games is crucial in prescribing and effectively manipulating training.

During basketball match-play, activity durations are highly variable given the frequent stoppages (e.g., free throws, time-outs) encountered. Additionally, these temporal sequences in activity requirements tend to occur randomly, as evidenced by very long periods (e.g., > 120 s) of continuous playing time with different stoppage durations (Salazar \& Castellano, 2020). Given this variability in activity duration and playing time, assessing intensity in basketball is necessary when monitoring external load as it is less dependent on duration than measures capturing total load (volume). However, quantifying only average intensity across total games fails to detect passages of higher physical demands known as most demanding scenarios (MDS) (Vázquez-Guerrero \& Garcia, 2020). Recent research in basketball demonstrated that when assessing the MDS during different time windows, players are exposed to intensities much greater than the intensities previously reported using game averages (Fox et al., 2020; Vázquez-Guerrero et al., 2020)

Several studies have assessed the physical demands encountered by basketball players across entire games (Russell et al., 2020). However, this approach does not give a complete picture of fluctuations in physical demands across games, such as during individual game quarters. In this regard, the knowledge of the physical demands throughout all quarters is essential in understanding how a player's physical performance fluctuates relative to the accumulation of playing minutes across games (Scanlan et al., 2015). Existing data have shown a significant decrease in high-intensity actions (Ben Abdelkrim et al., 2007; Reina et al., 2019), total distance and player load between Quarters 1 and 2 in professional basketball (García et al., 2020). This decrease in external load was also evident between Quarters 3 and 4 in semi-professional basketball (Scanlan et al., 2015) and between early periods and overtime, as evidenced by a reduction in player load and low- and medium-intensity accelerations (Scanlan et al., 2019). Consequently, the physical demands imposed on players appears to decrease with game progression in basketball. Given these load fluctuations between quarters, it is likely that differences between quarters may also be apparent when assessing MDS.

When assessing the physical demands encountered by players for the purpose of optimizing training prescription, previous research has also detailed the importance of assessing demands relative to playing positions (Svilar et al., 2018). Based on their anthropometric characteristics, playing area, and individual skills (Gryko et al., 2018), basketball players are commonly categorised as guard, forward, and centre positions. In this regard, differences in physical demands have been shown among playing positions during training (Svilar et al., 2018) and games (Salazar et al., 2020) in professional male basketball players. In addition, a recent study by Vazquez-Guerrero et al. (2020) showed that the MDS of game-play are position-dependent in elite U-18 basketball players. However, no such investigation has been conducted to determine whether differences in the MDS faced by different playing positions and across game quarters also appear at a professional level in basketball. As such, to ensure precise training prescription at the professional level, a separate investigation assessing the MDS of professional players across game quarters and concerning positional differences is warranted. Thus, the purpose of this study was to compare the MDS encountered by professional basketball players across game quarters and playing positions during official matchplay.

\section{Methods}

An observational design was used to compare the MDS of basketball match-play across game quarters and playing positions. Local positioning system data were collected from 11 competitive league games during the 2018-19 season, completed on the same official basketball court in similar environmental conditions. Players who were injured during the game or did not play a minimum total time of 5 min were excluded from the analysis of that game (Vázquez-Guerrero et al., 2019), resulting in a total of 1809 individual observations.

The ten professional male basketball players (mean \pm SD, age: $20.0 \pm 1.5 \mathrm{yr}$; height: $200.9 \pm 8.4 \mathrm{~cm}$; and body mass: $93.6 \pm 16.0 \mathrm{~kg}$ ) that participated in this investigation belonged to a reserve squad of a Spanish Euroleague team and competed in LEB Oro (Spanish second division). All players were categorized into one of three playing positions: guards, forwards, and centres. Match-play was conducted according to official FIBA rules. Ethics committee approval was not required because players were routinely monitored during all training sessions and games in the course of the competitive season (Winter \& Maughan, 2009). However, they agreed to participate by providing their written consent prior to the commencement of the research. Additionally, the league permits publication of these data, and the study fulfilled the provisions of the Declaration of Helsinki (Harriss \& Atkinson, 2015).

The team played one game a week, held between Friday and Sunday, after a standard 45-min warm-up consisting of dynamic stretching, specific mobility exercises and individual basketball-specific skills such as passing, shooting, and dribbling. Within each structured microcycle, the team usually rested the day after a game and completed three to four strength sessions and four to five basketball-specific training sessions before the game.

During match-play, all players were continuously monitored using a local positioning system (WIMU PRO ${ }^{\mathrm{ms}}$, RealTrack Systems S.L., Almería, Spain), although data were only included when players were competing on the court (e.g., time as substitutes or rest time between quarters was not included). This ultra-wideband system includes six antennas, which were placed in the form of a rectangle for better signal emission and reception (Figure 1). With a sampling frequency for positioning data of $18 \mathrm{~Hz}$, the local positioning system operates using triangulation between the antennas and the units (the six antennas send a signal to the units every 55.5 $\mathrm{ms}$ ). The device then calculates the time required to receive the signal and derives the unit position (coordinates $\mathrm{X}$ and 


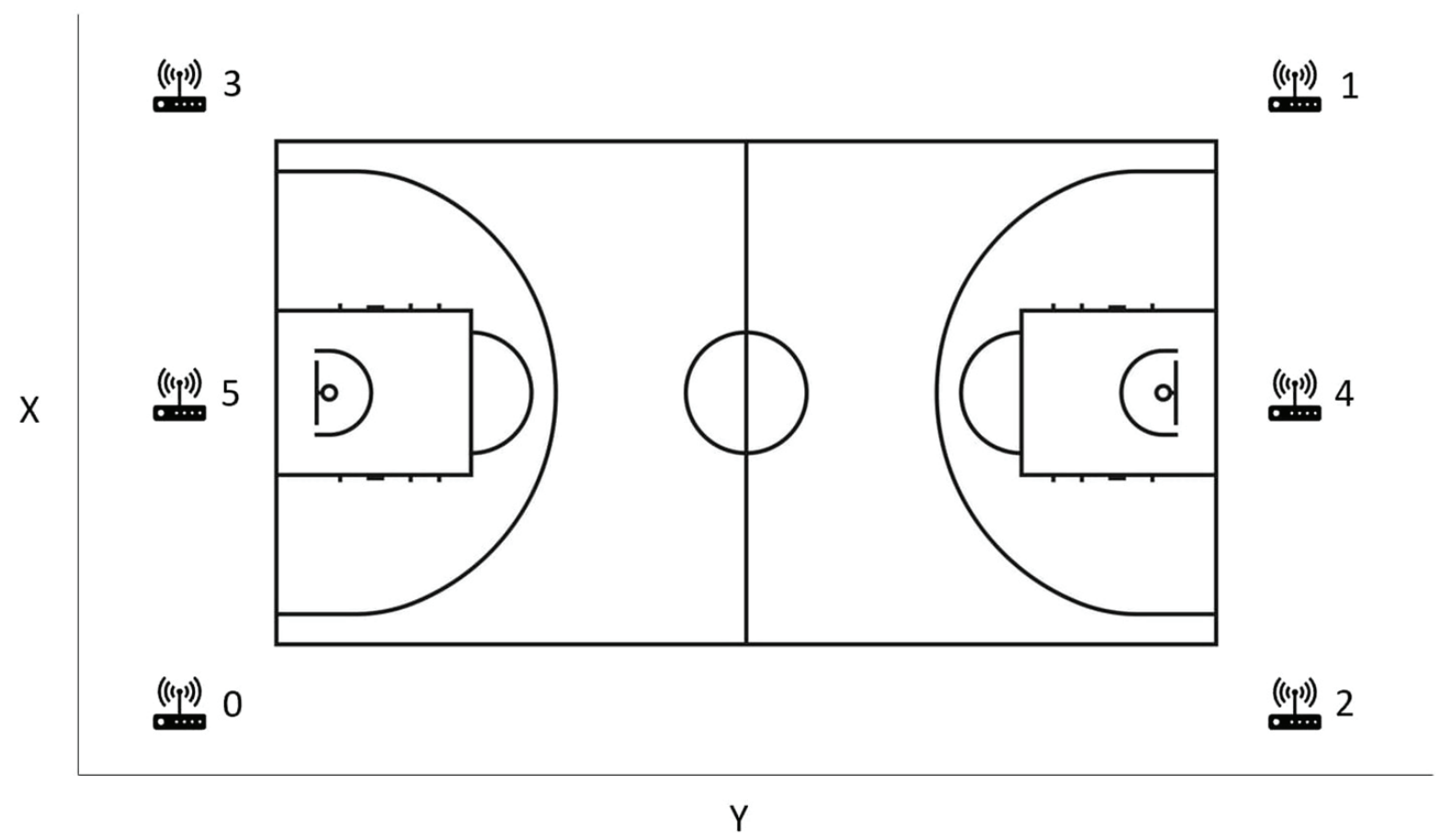

Figure 2. Ultra-wideband positioning system setup around the basketball court. $X$ is court width, $y$ is court length and $z$ is height of the antenna. Numbers show the disposition of antennas in $\mathrm{cm}$ : 0 is $x=0, y=0, z=600 ; 1$ is $x=2924, y=5208, z=600$; 2 is $x=0, y=5208, z=600 ; 3$ is $x=2928, y=7, z=600 ; 4$ is $x=1469, y=5207, z=600$; and 5 is $x=1456, y=2, z=600$

Y) using one of the antennas as a reference.

Based on the manufacturer's recommendations, the tracking units were placed in a custom-made vest located in the centre of the player's upper back using an adjustable harness (IMAX, Lleida, Spain). Players wore the same inertial unit and vest for each game across the season to reduce any potential between-device variability. WIMU PRO ${ }^{\mathrm{in}}$ has been shown to have good/acceptable accuracy and inter- and intra-unit reliability for ultra-wideband positioning (Bastida-Castillo et al., 2018, 2019). The system-specific SPRO ${ }^{\mathrm{m}}$ software (version 958, RealTrack Systems, Almería, Spain) was used to download and analyse the data on the physical demands.

Similar to previous research (Vázquez-Guerrero et al., 2020; Whitehead et al., 2018), the following physical demand parameters were measured: 1) Total distance $(\mathrm{m})$; 2) distance $>18 \mathrm{~km} \cdot \mathrm{h}^{-1}(\mathrm{~m}) ; 3$ ) distance $\left.>21 \mathrm{~km} \cdot \mathrm{h}^{-1}(\mathrm{~m}) ; 4\right)$ number of sprints $>18 \mathrm{~km} \cdot \mathrm{h}^{-1}$; 5) number of sprints $>21 \mathrm{~km} \cdot \mathrm{h}^{-1}$; 6) number of accelerations $>2 \mathrm{~m} \cdot \mathrm{s}^{-2} ; 7$ ) number of decelerations $\left.>2 \mathrm{~m} \cdot \mathrm{s}^{-2} ; 8\right)$ number of accelerations $>3 \mathrm{~m} \cdot \mathrm{s}^{-2}$; and 9) number of decelerations $>3 \mathrm{~m} \cdot \mathrm{s}^{-2}$. The distance was measured via positional differentiation (change in location with each signal), whereas acceleration and deceleration were calculated via double differentiation from the positional data recorded by the local positioning system (Malone et al., 2017). In line with Vázquez-Guerrero et al. (2020), the analysis of the MDS consisted of identifying the maximum values of the physical demand parameters of interest using a rolling average technique over five different periods $(30,60,120,180$ and 300 s). The 30 -s period was chosen because it represents the average duration of continuous playing before a stoppage is encountered in professional basketball, even though longer scenarios up to 120 -s are uncommon but possible (Salazar \& Castellano, 2020). In addition, 180- and 300-s periods were chosen as these durations reflect those often used by coach- es when prescribing training drills (Vázquez-Guerrero et al., 2020).

All data are presented as mean \pm standard deviation (SD). For each outcome measure, linear mixed models with Bonferroni post hoc tests were used to compare the MDS between game quarters for each positional group (guards, forwards, and centres). In the model, a game quarter was included as the fixed term (4 levels), and a participant was included as a random term to account for multiple data samples obtained for each participant (Peugh, 2010). Linear mixed models with Bonferroni post hoc tests were also used to compare the MDS between playing positions within each game quarter. In these analyses, a position was included as the fixed term (3 levels) and participant as a random term. Statistical significance was accepted where $\mathrm{P}<0.05$.

For all pairwise comparisons, Cohen's effect sizes with 95\% Confidence Intervals were computed and interpreted as trivial: $<0.2$, small: $0.2-0.59$, moderate: 0.6-1.19, large: $1.2-1.99$, and very large: $\geq 2$ (Hopkins, 2006). Statistical analyses and post hoc tests were conducted using the "lmerTest" and "emmeans" packages, respectively, on RStudio (Version 4.0.2), and effects sizes and confidence intervals were calculated using a customized Excel spreadsheet (Version 15, Microsoft Corporation, Redmond, USA).

\section{Results}

Descriptive statistics for the MDS within each quarter for each positional group are presented in Table 1. Results of the statistical analyses between game quarters in centres are presented in Table 2. Among centres, total distance was higher in Quarter 1 than Quarter 2 in the 120-s and 180-s periods and higher in Quarter 3 than Quarter 2 in the 180-s period $(\mathrm{P}<0.05)$. In addition, distance $>21 \mathrm{~km} \cdot \mathrm{h}^{-1}$ was higher during Quarter 1 compared to Quarter 2 in the 60-s and 180 -s periods $(\mathrm{P}<0.05)$. 


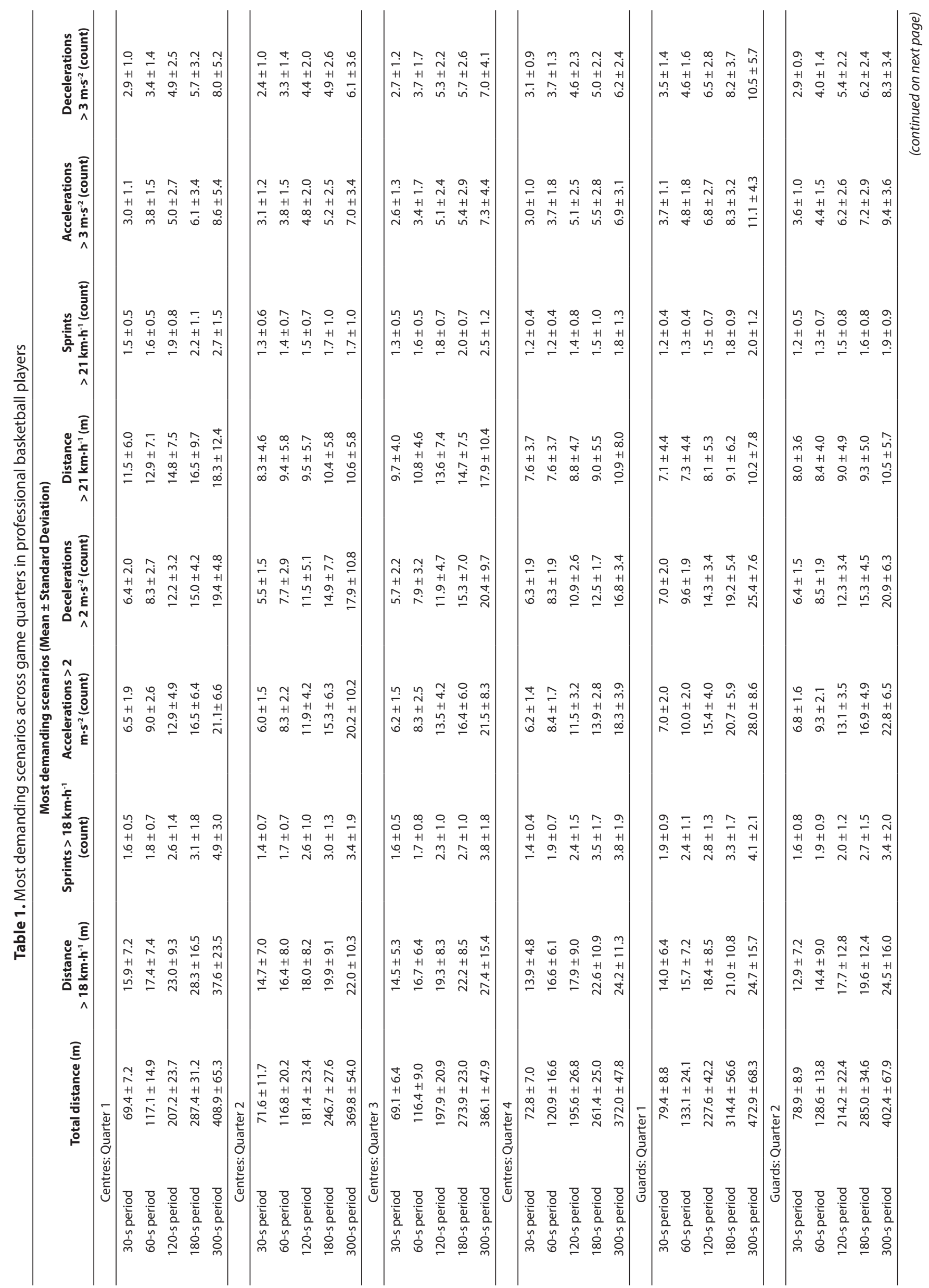




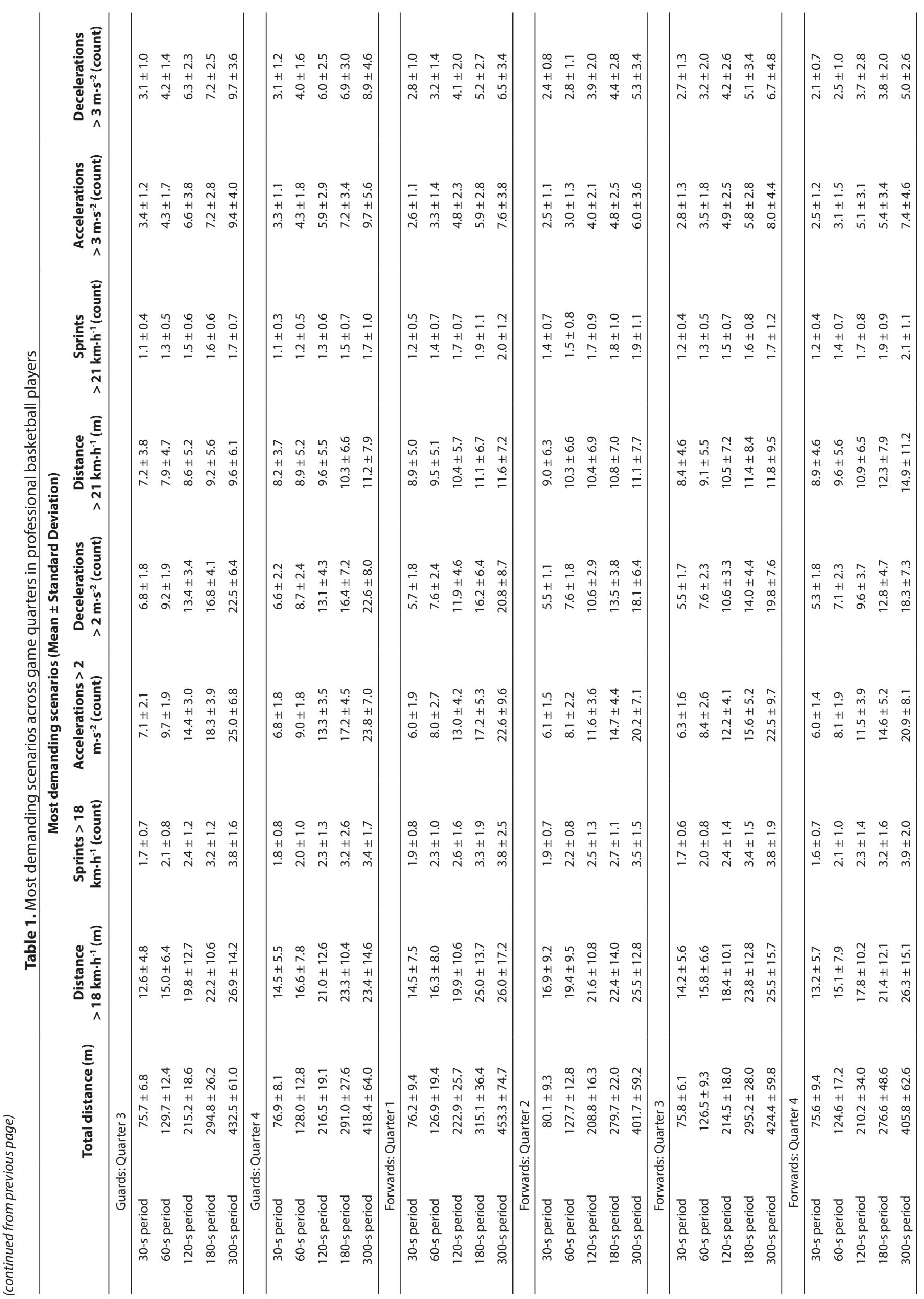




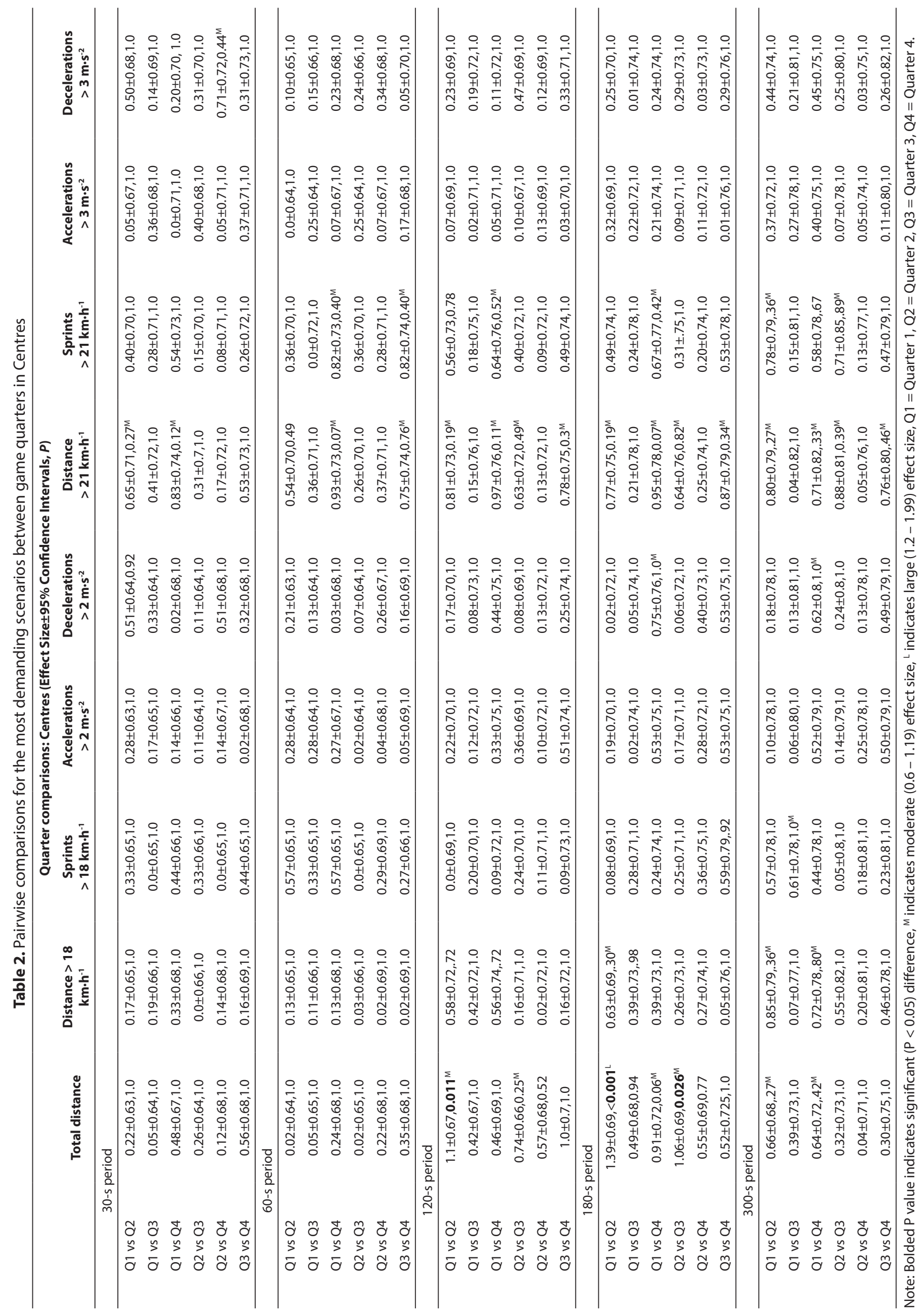




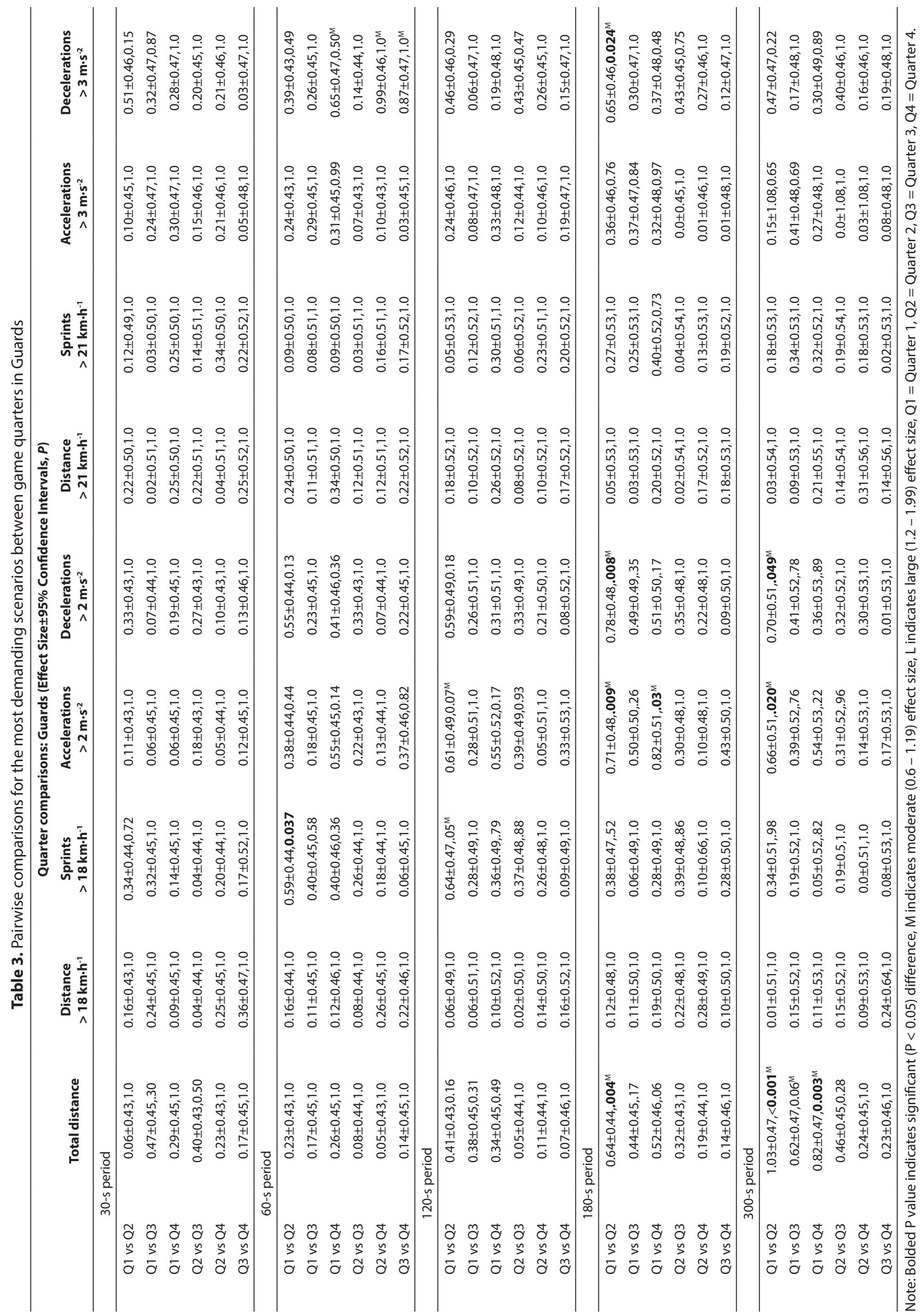




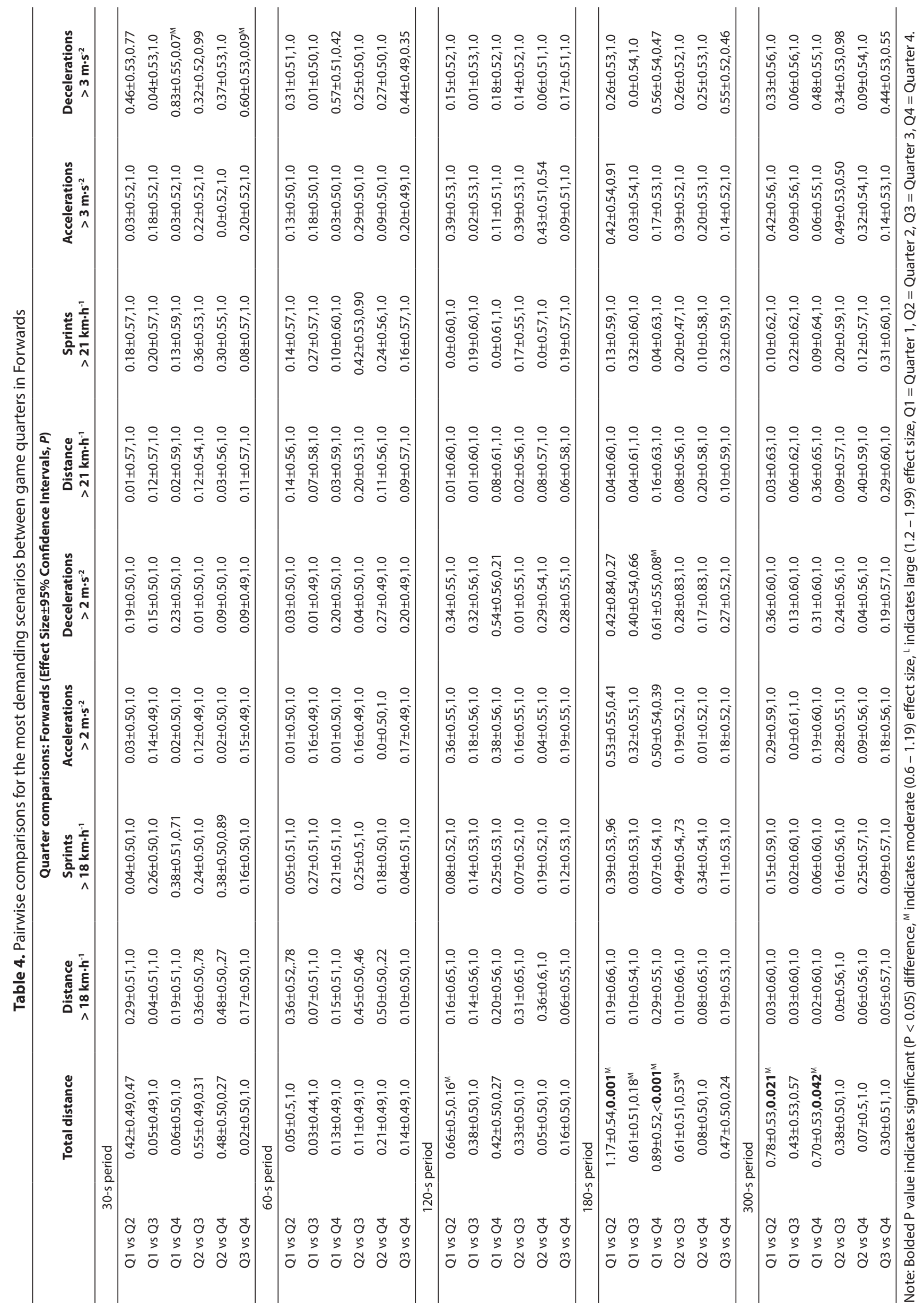




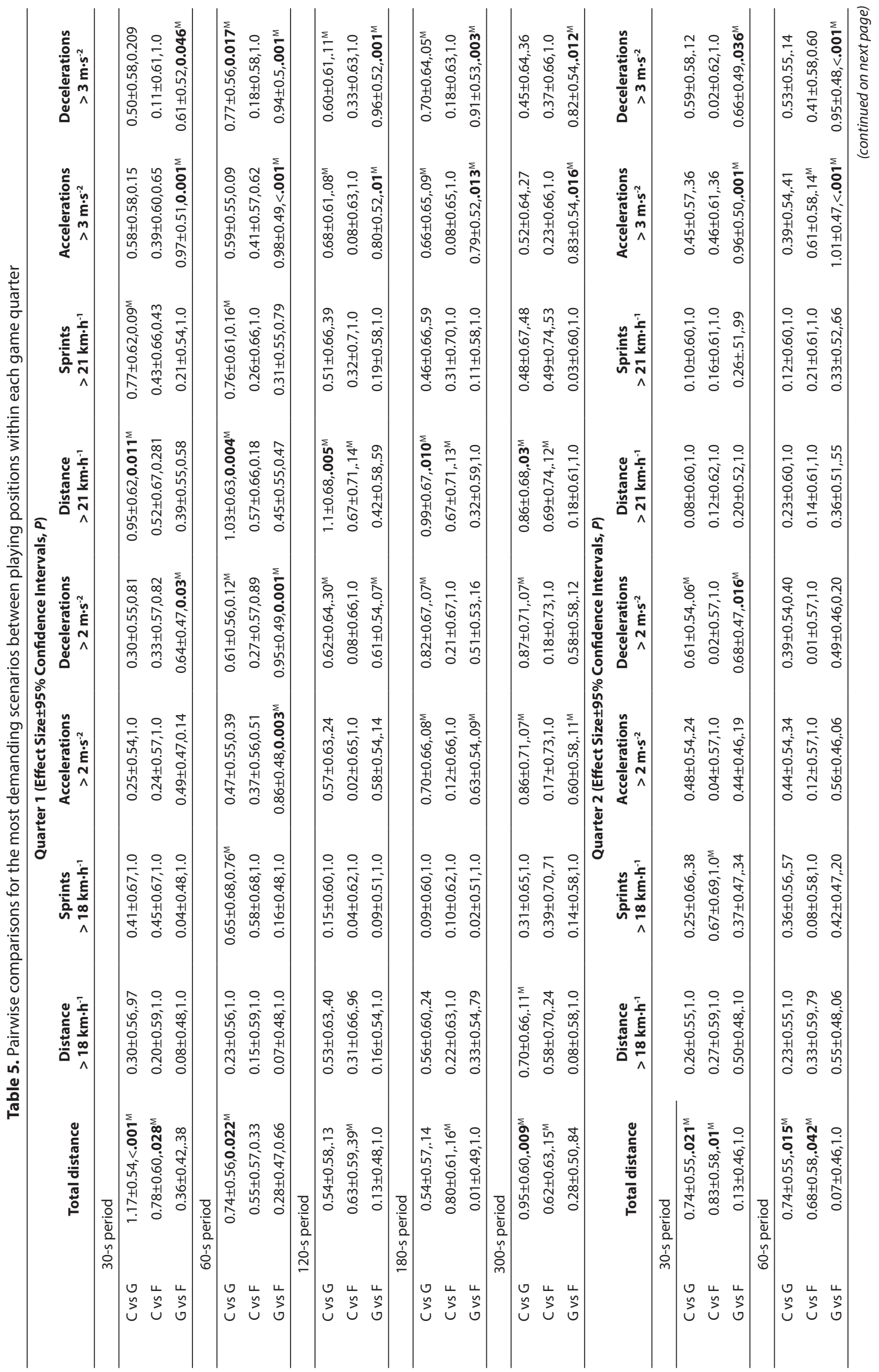




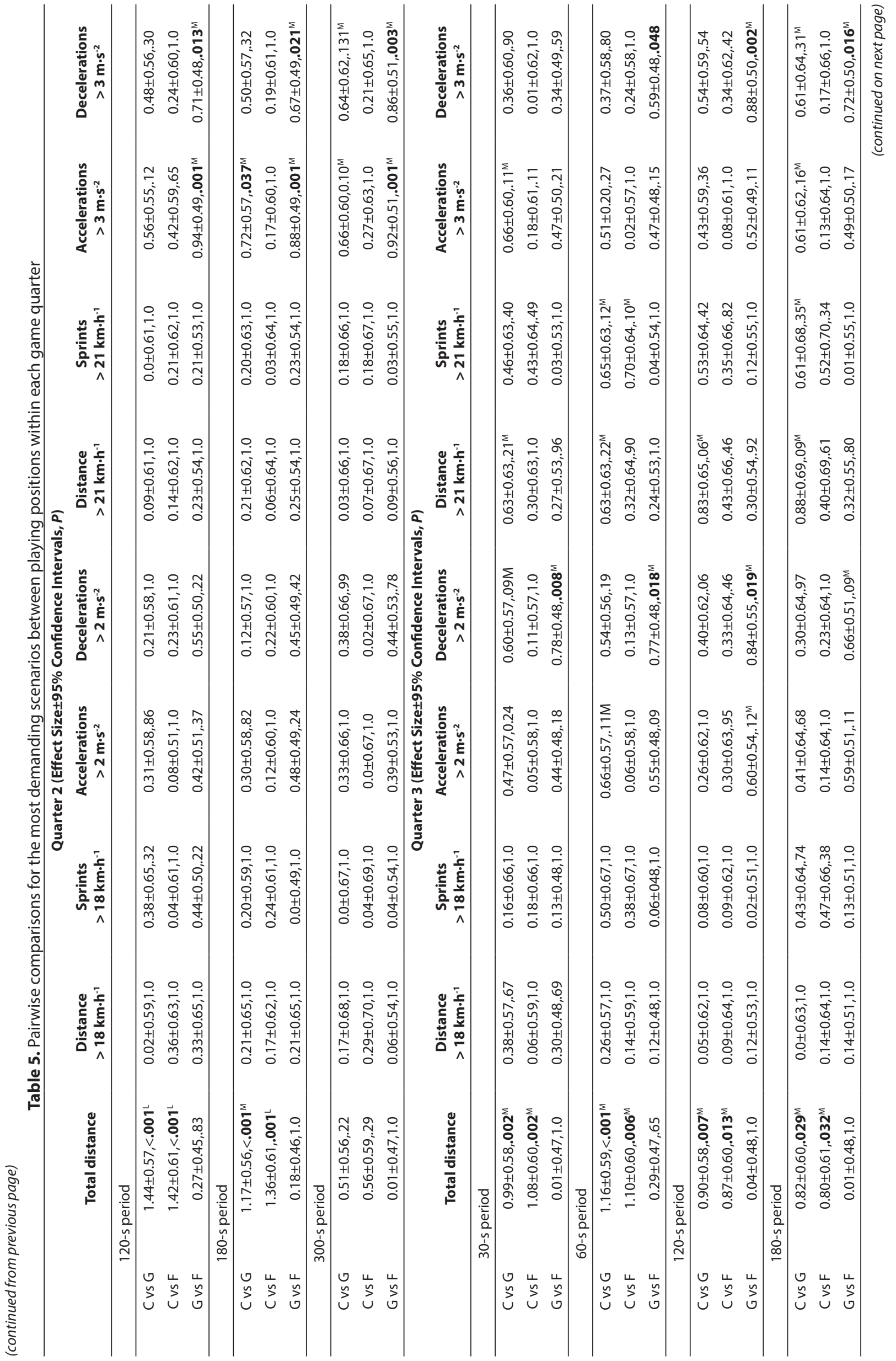




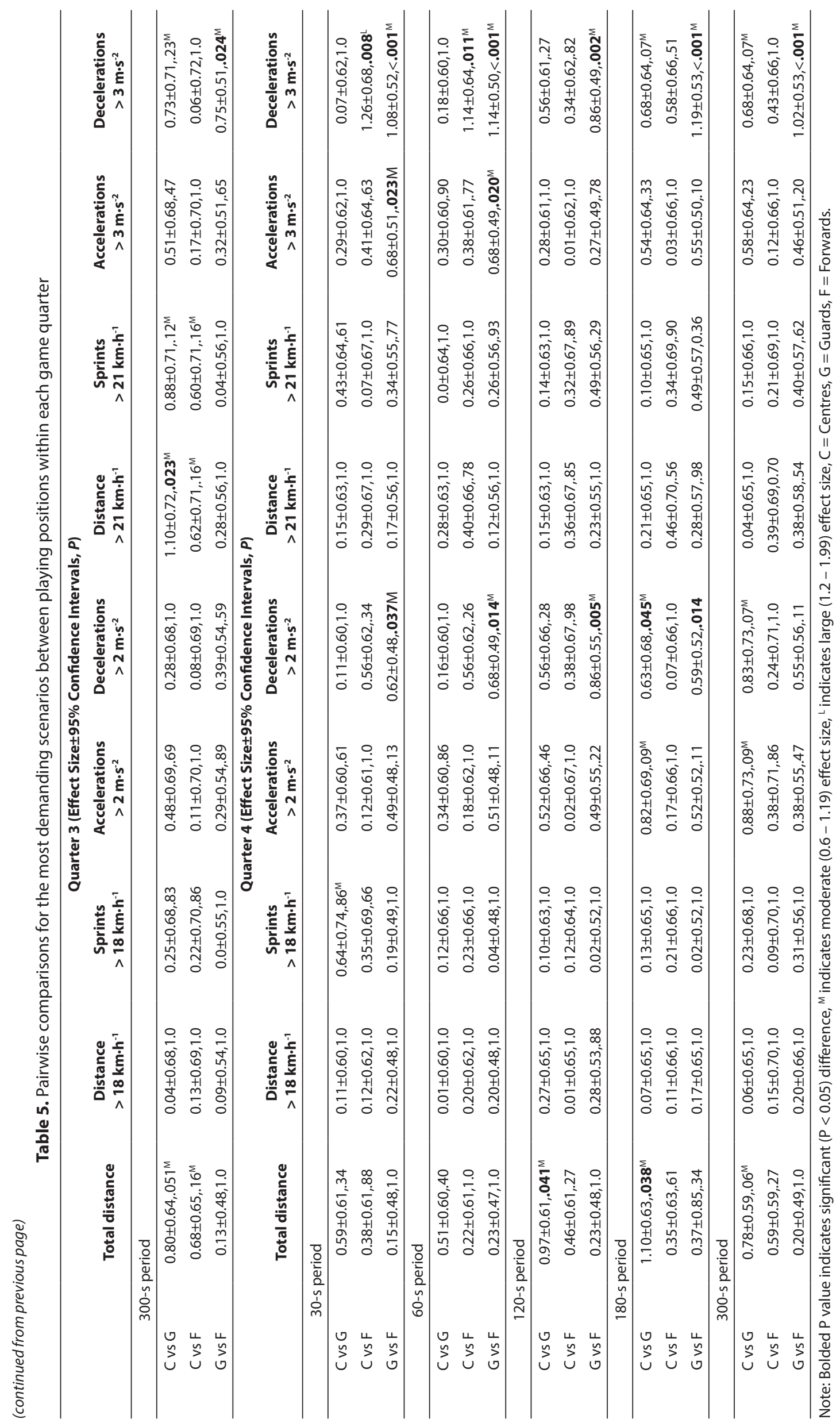


Results of the statistical analyses between game quarters in guards are presented in Table 3. In guards, total distance was higher in Quarter 1 than Quarter 2 in the 180-s and 300-s periods and higher in Quarter 1 than Quarter 4 in the 300-s period $(\mathrm{P}<0.05)$. The number of sprints $>18 \mathrm{~km} \cdot \mathrm{h}^{-1}$ was higher in Quarter 1 than Quarter 2 in the 60 -s period $(\mathrm{P}<0.05)$. Accelerations and decelerations $>2 \mathrm{~m} \cdot \mathrm{s}^{-2}$ were more frequent during Quarter 1 than Quarter 2 in the 180-s and 300-s periods, accelerations $>3 \mathrm{~m} \cdot \mathrm{s}^{-2}$ were more frequent during Quarter 1 than Quarter 4 in the 180 -s period, and accelerations $>3 \mathrm{~m} \cdot \mathrm{s}$ ${ }^{2}$ were more frequent during Quarter 1 than Quarter 2 in the 180 -s period $(\mathrm{P}<0.05)$.

Results of the statistical analyses between game quarters in forwards are presented in Table 4. In forwards, total distance was higher during Quarter 1 than Quarters 2 and 4 in the 180 -s and 300 -s periods $(\mathrm{P}<0.05)$. All other differences were non-significant $(\mathrm{P}>0.05)$, and the effect size magnitude ranged from trivial to moderate.

Results of the statistical analyses playing positions for each game quarter are presented in Table 5. In Quarter 1, total distance was higher in guards than centres in the 30-s, $60-s$, and 300-s periods and higher in forwards than centres in the 30 -s period $(\mathrm{P}<0.05)$. Accelerations and decelerations $>2 \mathrm{~m} \cdot \mathrm{s}^{-2}$ were more frequent in guards than forwards in the 60 -s periods, and decelerations $>2 \mathrm{~m} \cdot \mathrm{s}^{-2}$ were more frequent in guards than forwards in the 30 -s period $(\mathrm{P}<0.05)$. Distance $>21 \mathrm{~km} \cdot \mathrm{h}^{-1}$ was higher in centres than guards for all sample durations $(\mathrm{P}<0.05)$. Accelerations and decelerations $>3 \mathrm{~m} \cdot \mathrm{s}$ ${ }^{2}$ were more frequent in guards than forwards for all sample periods and more frequent in guards than centres in the 60-s period $(\mathrm{P}<0.05)$.

In Quarter 2, total distance was higher in centres than guards and higher in forwards than centres in the 30-s to 180 $\mathrm{s}$ periods, and accelerations $>2 \mathrm{~m} \cdot \mathrm{s}^{-2}$ were more frequent in guards than forwards in the 30 -s period $(\mathrm{P}<0.05)$. Accelerations and decelerations $>3 \mathrm{~m} \cdot \mathrm{s}^{-2}$ were more frequent in guards than forwards for all periods, and accelerations $>3 \mathrm{~m} \cdot \mathrm{s}^{-2}$ were more frequent in guards than centres in the 180 -s period ( $P$ $<0.05)$.

In Quarter 3, total distance was higher in guards than forwards and centres in the 30 -s to 180 -s periods $(\mathrm{P}<0.05)$. Decelerations $>2 \mathrm{~m} \cdot \mathrm{s}^{-2}$ were more frequent in guards than forwards in the $60-\mathrm{s}$ and 120 -s periods, and distance $>21 \mathrm{~km} \cdot \mathrm{h}$ ${ }^{1}$ was higher in centres than guards in the $300-s$ period ( $\mathrm{P}$ $<0.05$ ). Decelerations $>3 \mathrm{~m} \cdot \mathrm{s}^{-2}$ were more frequent in guards than forwards in the 60 -s to 300 -s periods $(\mathrm{P}<0.05)$.

In Quarter 4, the total distance was higher in guards than centres in the 120-s and 180-s periods. Decelerations $>2 \mathrm{~m} \cdot \mathrm{s}^{-2}$ were more frequent in guards than forwards in the 30-180-s periods and more frequent in guards than centres in the 180-s period $(\mathrm{P}<0.05)$. Accelerations $>3 \mathrm{~m} \cdot \mathrm{s}^{-2}$ were more frequent in guards than forwards in the 30 - and 60 -s periods $(\mathrm{P}<0.05)$. Decelerations $>3 \mathrm{~m} \cdot \mathrm{s}^{-2}$ were more frequent in guards than forwards for all sample periods and more frequent in centres than forwards in the 30- and 60-s periods $(\mathrm{P}<0.05)$. All other differences were non-significant and trivial-small in magnitude $(\mathrm{P}>0.05)$.

\section{Discussion}

In combination, the trends in our data support previous work in basketball highlighting reductions in MDS with game progression (Fox et al., 2020; Vázquez-Guerrero et al., 2020). A novel finding of this work is that in professional players, differences in MDS appear position-dependent and varied based on the external load variables and sample periods assessed.

Similar to previous research that used peak values to examine the differences between quarters (Fox et al., 2020; Vázquez-Guerrero et al., 2020), this investigation suggests that decreases in the MDS are evident across basketball games, with differences most prevalent between Quarters 1 and 2 over longer periods ( $\geq 120 \mathrm{~s}$ ) across all playing positions. Our findings also revealed more accelerations and decelerations $>2$ $\mathrm{m} \cdot \mathrm{s}^{-2}$ in the 180 and 300-s periods during Quarter 1 compared to Quarter 2, reflecting the data obtained in elite under-18 basketball players (Vázquez-Guerrero et al., 2020). Given that much of the physical stimulus imposed on basketball players is a result of intermittent, physically demanding movements, such as accelerations, decelerations, and change of direction (Stojanović et al., 2018), these variables are likely more sensitive to changes in external load, with respect to fluctuations in the MDS. Consequently, assessing high-intensity $\left(>2 \mathrm{~m} \cdot \mathrm{s}^{-}\right.$ ${ }^{2}$ ) accelerations when quantifying the MDS of training and match-play may be of particular importance to practitioners when prescribing and manipulating the external training load of players.

Although a trend emerged for MDS to decrease across the game, it is important to note that in some instances (e.g., distance $>18$ and $>21 \mathrm{~km} \cdot \mathrm{h}^{-1}$ for guards and forwards over varied sample periods), the highest MDS occurred in later game periods (Quarters 3 and 4). While past work has suggested that decreases in MDS may be related to fatigue-related mechanisms (Fox et al., 2020), the findings of this study suggest that it may be more closely related to outcomes such as tactical strategies and game-related contextual factors (e.g., level of opposition, score-line margin, win vs loss). As such, further research investigating the influence of tactics and contextual factors on MDS in basketball may be particularly useful in understanding potential mechanisms explaining fluctuations in MDS in basketball to further assist in more precise training prescription and manipulation.

When assessing the influence of playing position, the current investigation supports previous work (García et al., 2020; Vázquez-Guerrero et al., 2020), demonstrating position-dependent external load profiles in basketball. For instance, total distance was significantly lower in centres than guards and forwards during different periods in all four quarters. These results may partly be explained by a combination of technical and tactical profiles along with the anthropometric characteristics of players. Specifically, centres are required to play near the three-second zone, set screens and rebound during set-pieces (Sampaio et al., 2006) and are usually the tallest and heaviest players (Gryko et al., 2018), making them suited to positions with lower movement demands.

Accelerations and decelerations $>2$ and $>3 \mathrm{~m} \cdot \mathrm{s}^{-2}$ also presented great variation between playing positions. Specifically, guards completed more accelerations and decelerations during all four quarters, presumably because they are required to perform a great number of intermittent, high-intensity movements (e.g., changes of direction) in half and full-court situations (e.g., cutting, perimeter play, defence, etc.). In interpreting this finding, it is also important to consider that some differences in MDS detected in the $>3 \mathrm{~m} \cdot \mathrm{s}^{-2}$ were not apparent when assessing the number of accelerations and decelerations $>2 \mathrm{~m} \cdot \mathrm{s}^{-2}$. Given that elite under-18 male basketball players 
have performed peak accelerations up to $3.6 \mathrm{~m} \cdot \mathrm{s}^{-2}$ during official games (Vázquez-Guerrero, Jones, et al., 2019), it is possible that the $2 \mathrm{~m} \cdot \mathrm{s}^{-2}$ threshold typically utilised does not reflect a true high-intensity effort when assessing accelerations and decelerations. Moreover, in basketball, the cut point used to assess "high-intensity" activity using inertial sensors appears to be somewhat arbitrary and often comes from the sensors' proprietary software rather than an evidence-based approach. As such, further work assessing the appropriateness of varied cut points to discriminate between intensities of accelerations and decelerations in basketball appears particularly valuable.

In conclusion, this study showed that the MDS of basketball match-play fluctuates across game quarters and varies between playing positions in professional players. Nevertheless, when interpreting the findings of this study, some notable limitations should be acknowledged. The small sample per position might limit the representativeness of the external loads encountered within each positional group. Finally, only the MDS of different physical demand parameters were measured for each player in isolation which does not take into account important contextual factors, such as activities completed by team-mates and opponents within the same game period as well as outcomes relating to tactical strategies, score-line margin, and the game results (e.g., whether the team was winning or losing). Therefore, future research should expand the analysis of the MDS during different competition formats (e.g., pre-season, tournament, play-off) while considering game-related contextual factors (e.g., offence, defence, transitions, score) to understand fluctuations in MDS of basketball matchplay better and consequently optimise training prescription and player performance.

\section{Acknowledgements}

The authors of this article would like to thank the professional basketball players who took part in this study and all the Futbol Club Barcelona performance department staff members who assisted in player monitoring. The authors also gratefully acknowledge the support of "Barça Innovation Hub" for their help and collaboration concerning the practice of qualitative research.

\section{References}

Bastida-Castillo, A., Gómez Carmona, C. D., De la Cruz Sánchez, E., \& Pino Ortega, J. (2018). Accuracy, intra- and inter-unit reliability, and comparison between GPS and UWB-based position-tracking systems used for timemotion analyses in soccer. European Journal of Sport Science, 18(4), 450-457. https://doi.org/10.1080/1746139 1.2018 .1427796

Bastida-Castillo, A., Gómez-Carmona, C., De la CruzSánchez, E., Reche-Royo, X., Ibáñez, S., \& Pino Ortega, J. (2019). Accuracy and inter-unit reliability of ultra-wideband tracking system in indoor exercise. Applied Sciences, 9(5), 939. https://doi.org/10.3390/app9050939

Ben Abdelkrim, N., El Fazaa, S., \& El Ati, J. (2007). Timemotion analysis and physiological data of elite under-19year-old basketball players during competition. British Journal of Sports Medicine, 41(2), 69-75. https://doi. org/10.1136/bjsm.2006.032318

Fox, J. L., Conte, D., Stanton, R., McLean, B., \& Scanlan, A. T. (2020). The application of accelerometer-derived moving averages to quantify peak demands in basketball: A comparison of sample duration, playing role, and session type. Journal of Strength and Conditioning Research, 0-0. https://doi.org/10.4018/978-1-59904-828-4.chtpg

Fox, J. L., Salazar, H., García, F., \& Scanlan, A. T. (2020). Peak external intensity decreases across quarters during basketball games. Montenegrin Journal of Sports Science and Medicine, 8(2), 5-12. https://doi.org/10.26773/ mjssm.210304

García, F., Vázquez-Guerrero, J., Castellano, J., Casals, M., \& Schelling, X. (2020). Differences in physical demands between game quarters and playing positions on professional basketball players during official competition. Journal of Sports Science and Medicine, 19(2), 256-263.

Gryko, K., Kopiczko, A., Mikołajec, K., Stasny, P., \& Musalek, M. (2018). Anthropometric Variables and Somatotype of Young and Professional Male Basketball Players. Sports, 6(1), 9. https://doi.org/10.3390/sports6010009

Harriss, D. J., \& Atkinson, G. (2015). Ethical standards in sport and exercise science research: 2016 update. International Journal of Sports Medicine, 36(14), 1121-1124. https://doi. org/10.1055/s-0035-1565186

Hopkins, W. G. (2006). A scale of magnitudes for effect statistics. SportSci. http://www.sportsci.org/resource/stats/ index.html\%0A

Malone, J. J., Lovell, R., Varley, M. C., \& Coutts, A. J. (2017). Unpacking the black box: Applications and considerations for using gps devices in sport. International Journal of Sports Physiology and Performance, 12, 18-26. https://doi. org/10.1123/ijspp.2016-0236

Narazaki, K., Berg, K., Stergiou, N., \& Chen, B. (2009). Physiological demands of competitive basketball. Scandinavian Journal of Medicine and Science in Sports, 19(3), 425-432. https://doi.org/10.1111/j.16000838.2008.00789.x

Peugh, J. L. (2010). A practical guide to multilevel modeling. Journal of School Psychology, 48(1), 85-112. https://doi. org/10.1016/j.jsp.2009.09.002

Reina, M., García-Rubio, J., Pino-Ortega, J., \& Ibáñez, S. J. (2019). The acceleration and deceleration profiles of U-18 women's basketball players during competitive matches. Sports, 7(7), 165. https://doi.org/10.3390/sports7070165

Russell, J. L., Mclean, B. D., Impellizzeri, F. M., Strack, D. S., \& Coutts, A. J. (2020). Measuring physical demands in basketball: an explorative systematic review of practices. In Sports Medicine (Issue November). Springer International Publishing. https://doi.org/10.1007/s40279-020-01375-9

Salazar, H., \& Castellano, J. (2020). Analysis of basketball game: relationship between live actions and stoppages in different levels of competition. E-Balonmano.Com: Revista de Ciencias Del Deporte, 16, 109-118.

Salazar, H., Castellano, J., \& Svilar, L. (2020). Differences in external load variables between playing positions in elite basketball match-play. Journal of Human Kinetics, 75, 131. https://doi.org/DOI: 10.2478/hukin-2020-0054 DOI: 10.2478/hukin-2020-0054

Sampaio, J., Janeira, M., Ibáñez, S., \& Lorenzo, A. (2006). Discriminant analysis of game-related statistics between basketballguards, forwards and centres in three professional leagues. European Journal of Sport Science, 6(3), 173-178. https://doi.org/10.1080/17461390600676200

Sansone, P., Tschan, H., Foster, C., \& Tessitore, A. (2020). Monitoring training load and perceived recovery in female 
basketball: implications for training design. Journal of Strength and Conditioning Research, 34(10), 2929-2936. https://doi.org/10.1519/JSC.0000000000002971

Scanlan, A. T., Stanton, R., Sargent, C., O'Grady, C., Lastella, M., \& Fox, J. L. (2019). Working overtime: the effects of overtime periods on game demands in basketball players. International Journal of Sports Physiology and Performance, 1, 1-20. https://doi.org/10.1123/ijspp.2018-0906

Scanlan, A. T., Tucker, P. S., Dascombe, B. J., \& Berkelmans, D. M. (2015). Fluctuations in activity demands across game quarters in professional and semi-professional male basketball. Journal of Strength \& Conditioning Research, 29(11), 3006-3015. https://doi.org/https://doi. org/10.1519/JSC.0000000000000967

Stojanović, E., Stojiliković, N., Scanlan, A. T., Dalbo, V. J., Berkelmans, D. M., \& Milanović, Z. (2018). The activity demands and physiological responses encountered during basketball match-play: a systematic review. Sports Medicine, 48(1), 111-135. https://doi.org/10.1007/s40279017-0794-Z

Svilar, L., Castellano, J., Jukic, I., \& Casamichana, D. (2018). Positional differences in elite basketball: selecting appropriate training. International Journal of Sports Physiology and Performance, 13(7), 947-952.

Vázquez-Guerrero, J., \& Garcia, F. (2020). Is it enough to use the traditional approach based on average values for basketball physical performance analysis? European Journal of Sport Science, 1-18. https://doi.org/10.1080/17 461391.2020.1838618

Vázquez-Guerrero, J., Ayala Rodríguez, F., García, F., \& Sampaio, J. E. (2020). The most demanding scenarios of play in basketball competition from elite Under-18 teams. Frontiers in Psychology, 11, 552. https://doi.org/https://doi. org/10.3389/fpsyg.2020.00552

Vázquez-Guerrero, J., Fernández-Valdés, B., Jones, B., Moras, G., Reche, X., \& Sampaio, J. (2019). Changes in physical demands between game quarters of U18 elite official basketball games. Plos One, 14(9), 1-14. https://doi.org/ https://doi.org/10.1371/journal.pone.0221818

Vázquez-Guerrero, J., Jones, B., Fernández-Valdés, B., Moras, G., Reche, X., \& Sampaio, J. (2019). Physical demands of elite basketball during an official U18 international tournament. Journal of Sport Sciences, 37(22), 2530-2537. https://doi.org/10.1080/02640414.2019.1647033

Whitehead, S., Till, K., Weaving, D., \& Jones, B. (2018). The use of microtechnology to quantify the peak match demands of the football codes: a systematic review. Sports Medicine, 48(11), 2549-2575. https://doi.org/10.1007/ s40279-018-0965-6

Winter, E. M., \& Maughan, R. J. (2009). Requirements for ethics approvals. Journal of Sports Sciences, 27(10), 985-985. https://doi.org/10.1080/02640410903178344 